\title{
CAPÍTULO IXX
}

\section{IMPACTO DE LA CULTURA DE INNOVACIÓN EN LAS MYPES DE VARIOS MUNICIPIOS DE LA ZONA METROPOLITANA LAJA-BAJÍO}

\author{
Carlos Alberto Rodríguez Rodríguez \\ Doctor en administración y gestión empresarial. Profesor. Universidad \\ Politécnica de Guanajuato, Cortazar, Gto. Correo electrónico: carodriguezr@ \\ upgto.edu.mx.
}

\author{
Roberto Godínez López \\ Doctor en administración. Director de la carrera de Administración. Universidad \\ de Guanajuato. Campus Celaya-Salvatierra. Correo electrónico: godinez.r@ \\ ugto.mx.
}

\section{Lidia Ramírez Lemus \\ Doctora en Educación. Profesor de tiempo completo en la Universidad Tecnológica del Suroeste de Guanajuato. Correo electrónico: Lramirez@utsoe. edu.mx.}

\section{Resumen}

Los tiempos actuales demandan empresas adaptables y competitivas debido a los altos niveles de exigencia en la economía moderna. La gestión del conocimiento se ha convertido en un factor fundamental para el sostenimiento de dichas organizaciones. La cultura de innovación está considerada por la literatura científica como un factor esencial para lograr ventajas competitivas empresariales sostenibles. Las micro y pequeñas empresas (MYPES) son el motor de la economía de muchos países del mundo incluyendo México. El estado de Guanajuato es el sexto lugar de los estados del país que cuenta con el mayor número de MIPYMES, manifestando ello la importancia que tienen estas empresas para dicha entidad. El objetivo del presente estudio es caracterizar la cultura de innovación de las MYPES de los municipios de Celaya, Apaseo el alto, Apaseo el grande y Cortazar, los cuales pertenecen a la zona metropolitana Laja-Bajío. El diseño de investigación es cuantitativo, no experimental con alcance descriptivo. La muestra se calculó utilizando algoritmos para poblaciones infinitas y se obtuvo a través de la técnica de muestro no probabilístico por conveniencia. En los resultados de investigación se logró encontrar evidencia estadística que indica que existen dentro de las empresas estudiadas rasgos de cultura de innovación, tales como la apertura de los lideres a aceptar nuevas ideas, interés del personal a generar ideas innovadoras, planes de incentivos parcialmente implementados, uso de medios electrónicos para mantenerse informado sobre la situación de los mercados, análisis de los productos del mercado para detectar necesidades, e innovación, entre otras. Además, se demostró que los municipios que presentan mejores desempeños en características de cultura de 
innovación son Apaseo el grande seguido por Celaya. La presente investigación dará la pauta para realizar estudios futuros que generen información para contribuir a resolver las múltiples problemáticas que presentan este tipo de organizaciones.

Palabras clave: cultura, innovación, Guanajuato, microempresas.

\title{
IMPACT OF THE CULTURE OF INNOVATION IN THE MYPES OF SEVERAL MUNICIPALITIES IN THE METROPOLITAN AREA LAJA-BAJÍO
}

\begin{abstract}
The current times demand adaptable and competitive companies due to the high levels of demand in the modern economy. Knowledge management has become a fundamental factor in sustaining these organizations. The culture of innovation is considered by the scientific literature as an essential factor to achieve sustainable competitive business advantages. Micro and small businesses (MYPES) are the engine of the economy of many countries of the world including Mexico. The state of Guanajuato is the sixth place in the states of the country that has the largest number of MSMEs, stating the importance of these companies for that entity. The objective of this study is to characterize the innovation culture of the MYPES of the municipalities of Celaya, Apaseo el alto, Apaseo el grande and Cortazar, which belong to the metropolitan area Laja-Bajío. The research design is quantitative, not experimental with descriptive scope. The sample was calculated using algorithms for infinite populations and was obtained through the nonprobabilistic sampling technique for convenience. In the research results it was possible to find statistical evidence that indicates that within the companies studied features of innovation culture, such as the opening of leaders to accept new ideas, interest of staff to generate innovative ideas, partially implemented incentive plans, use of electronic means to keep informed about the situation of the markets, analysis of market products to detect needs, and innovation, among others. In addition, it was shown that the municipalities with the best performance in innovation culture characteristics are Apaseo el grande followed by Celaya. This research will set the tone for future studies that generate information to help solve the multiple problems presented by this type of organizations.
\end{abstract}

Keywords: culture, innovation, Guanajuato, microenterprises.

\section{Introducción}

Vivimos en la era de la información donde la gestión del conocimiento se vuelve un factor fundamental en el sostenimiento y desarrollo de las organizaciones. La innovación es consecuencia del conocimiento, genera diferenciación y ellos son factores de competitividad, los cuales se vuelven cada vez más importantes debido a la situación de 
crisis económica contemporánea. De acuerdo a Islam y Tedford, (2012), en el ambiente de negocios altamente competitivo en el que viven las organizaciones se encuentran bajo demasiada presión debido a la economía de libre mercado, rápidos desarrollos tecnológicos y cambios continuos en la demanda de los clientes. Para hacer frente a dichas tendencias de negocios, las demandas en los sistemas modernos de manufactura han requerido incrementar la flexibilidad, mayores estándares de calidad y capacidades de innovación.

Por lo tanto, las organizaciones deben adaptarse lo más rápidamente posible a los cambios que el entorno les exige. La capacidad para innovar depende de aspectos como la organización y los sistemas, además del vínculo entre las nuevas tecnologías existentes $\mathrm{y}$ el aprendizaje organizacional traducido en personal preparado, con experiencia y creatividad.

\section{Problema de investigación}

Para Gil y Jiménez (2014, p.165) “En la región de América Latina, cerca de 70\% del producto interno bruto (PIB) proviene de grandes empresas, mientras que en la Organización para la Cooperación y Desarrollo Económico (OCDE) aproximadamente el $40 \%$ de la producción es generada por estas empresas y el resto por las PYME. El elevado aporte al empleo combinado con el bajo aporte a la producción que caracteriza a las PYMES en América Latina es reflejo de la estructura productiva heterogénea, la especialización en productos de bajo valor agregado y su reducida participación en las exportaciones, inferior a 5\% en la mayoría de los países. El resultado es que la brecha de productividad entre los países de la región y los de la OCDE tiende a persistir en el tiempo".

El estudio de las MYPES no solo contribuye a mejorar el desempeño de dichas empresas, sino a promover aspectos de cultura organizacional y de innovación que generen incrementos en la calidad, productividad, por tanto en la competitividad de las organizaciones así como de la sociedad en su conjunto, el traer mayor bienestar económico a las familias de esas empresas contribuye también al bienestar y estabilidad psicológica y social de la región y del país que tanto lo necesita en estos momentos de incertidumbre y agitación política, situación que redunda en beneficio de todos. 
El estado de Guanajuato se encuentra en el sexto lugar de los estados del país que cuenta con el mayor número de micros, pequeñas y medianas empresas (MIPYMES), hablando con esto de la importancia que debe tener este tipo de empresas para el Estado (Góngora, 2013). En un estudio realizado en las MIPYMES de Celaya, Guanajuato, los resultados evidenciaron la existencia de diez problemas principales, tales como:

Recursos financieros limitados, alto nivel de endeudamiento, cartera vencida, cambios en el costo de materia prima, bajo nivel de ventas, falta de capital para invertir, dificultad para adquirir préstamos, falta de capital de trabajo, ausencia de control financiero y de personal especializado (Maldonado, Ojeda, Uc, Valdez y Medina, 2017, p.250).

Lo anterior sirve de planteamiento de la importancia del estudio y análisis de los factores que inciden directamente en la productividad y competitividad, tales como los señalados anteriormente, la falta del uso de tecnología, la capacitación, la falta de acceso a créditos, la falta del desarrollo de una cultura de innovación. Dando como resultado el objetivo de la presente investigación, el cual es identificar las características de la cultura de innovación de las MYPES de los municipios de Celaya, Apaseo el Grande, Apaseo el Alto y Cortazar de la Zona metropolitana Laja - Bajío.

\section{Marco teórico}

\section{Región Laja-Bajío}

La región Laja-Bajío está conformada por ocho municipios Apaseo el Alto, Apaseo el Grande, Celaya, Comonfort, Cortazar, Santa Cruz de Juventino Rosas, Tarimoro y Villagrán. Sin embargo, por un decreto gubernamental se añadió al municipio de Jaral del Progreso (Periódico Oficial del Gobierno del Estado de Guanajuato, 2010) y en algunos estudios incluyen a los municipios colindantes en el Sur: Acámbaro, Coroneo, Jerécuaro, Salvatierra y Tarandacuao e incluso se llega a considerar a Salamanca en el Norponiente (Molina y Contreras, 2016).

\section{Definición de PYMES}

Para Rodríguez (2010), la definición relativa a las PYMES es: "aquella que posee el diseño en plena libertad, dirigida autónomamente y que no es dominante en la rama que opera”. 


\section{Cultura organizacional}

De acuerdo a Robbins (2014, p.51), la cultura organizacional se describe como los "valores, principios, tradiciones y formas de hacer las cosas que comparten los miembros de la empresa, influyen en la manera en que éstos actúan y distinguen a la organización entre todas las demás”.

\section{Innovación}

La conceptualización de la innovación ha tenido varias transformaciones y visiones a través del tiempo. Sampedro y Díaz (2016), mencionan que los países más ricos en la actualidad, tales como Estados Unidos, Europa Occidental y Japón son relativamente más innovadores.

Gomes \& Wojahn (2017), consideran que la innovación está íntimamente asociada a la capacidad de aprendizaje que poseen las organizaciones. El aprendizaje organizacional es considerado como uno de los factores precursores y con un efecto positivo en la innovación. La innovación supone novedad y puede generar resultados directos en el desempeño organizacional y de este modo asociarse como desempeño innovador, es decir, en los resultados derivados de la innovación logrando ventajas competitivas sostenibles.

Guerra (2010, p 247), menciona que... “La innovación es un cambio en el método o en la tecnología; una desviación útil y positiva de la forma como se hacían las cosas previamente" también se refiere a "la introducción de nuevos productos (ya sean bienes o servicios) o a su mejoramiento; también se refiere a nuevos procesos, formas de comercialización y mejoras organizacionales en las empresas”.

\section{Cultura de innovación}

De acuerdo a Carbache, Frías, Gutiérrez y Véliz (2017), se ha demostrado empíricamente que existe una fuerte correlación entre la cultura organizacional y la innovación, y esta última trae como consecuencia mayores niveles de productividad por parte del personal. De este modo, la cultura organizacional influye en el comportamiento 
de los trabajadores y puede incentivar o frenar la innovación, por tanto la productividad y competitividad en las organizaciones.

Robbins (2014), menciona que una cultura de innovación posee características tales como: desafío e involucramiento de los empleados con los objetivos organizacionales; libertad de los empleados para realizar sus actividades, tomar la iniciativa y expresar sus opiniones e ideas, confianza y disposición a apoyar a otros compañeros, tiempo de reflexión para elaborar nuevas ideas, alegría/buen humor en un ambiente espontaneo, resolución de conflictos a través de la participación en la toma de decisiones, toma de riesgos apoyado por la tolerancia de la gerencia ante la incertidumbre y la ambigüedad.

Cuando se desarrolla una cultura organizacional fuerte, se considera como una herramienta estratégica se genera competitividad en los negocios. Los líderes deben motivar a sus organizaciones a ser más innovadoras, sin embargo, los grandes retos están en sostener la competitividad de las empresas a través del tiempo (Putthiwanit, 2015).

Respecto de lo anterior, algunas investigaciones en regiones de la periferia de Colombia sugieren una correlación positiva directa entre la innovación y el capital intelectual en las organizaciones, que potencia la generación de niveles elevados de competitividad en las organizaciones. Debido a ello es recomendable que los gerentes entiendan que la clave de las ventajas competitivas se da en torno a la gestión del conocimiento, involucrando al talento humano, a través de soportes institucionales y relacionales (Andrade, Gasca, Perdomo, Benavides. y Sánchez, 2019).

Un ejemplo de innovación empresarial lo encontramos en la empresa Chedraui en la cual la aplicación de tecnología de la información resultó en la disminución de costos de operación, inventarios, procesamientos de órdenes de compra, distribución de mercancías y reducción de tiempo de las tareas administrativas. Lo que lograron hacer los líderes del corporativo de tiendas Chedraui fue aprovechar las últimas innovaciones tecnológicas, como fue el caso del Sistema, Aplicaciones y Procesos SAP, lo cual mejoró el control administrativo y organizacional en sus tiendas, agilizando las actividades diarias y reduciendo los costos de operación e inventarios. (Torres, González y Cano, 2019). 
Rueda y Rodenes (2010), consideran que para generar una cultura innovadora que pueda mantenerse a través del tiempo las organizaciones deben reunir una serie de características culturales orientadas hacia la innovación. En la figura 1, se muestran dichos factores.

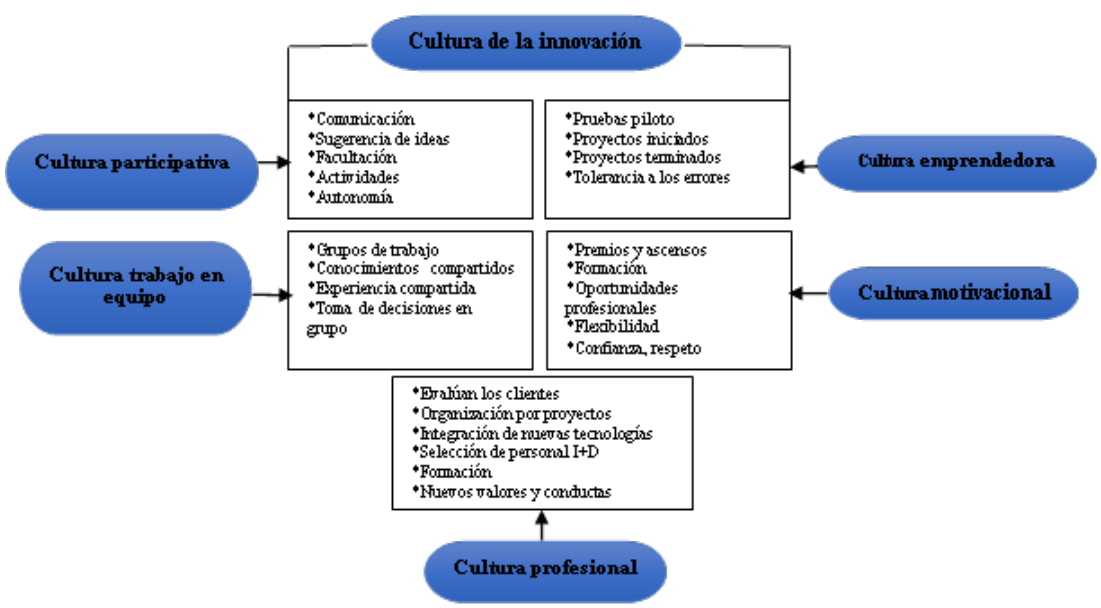

Figura 1. Dimensiones de la cultura orientadas a la innovación. Recuperada de “Hacia una cultura innovadora en las empresas,” de G.E. Rueda y M. Rodenes, 2010, Universidad Pontificia Bolivariana. Universitat Politècnica de València. p. 4.

\section{Método}

Se trata de una investigación cuantitativa, en donde el diseño de investigación que se utiliza es del tipo no experimental, siendo su alcance del tipo descriptivo con la finalidad de analizar el fenómeno de la cultura de innovación en las MYPES de municipios pertenecientes a la Zona Metropolitana Laja Bajío (Hernández Sampieri, FernandezCollado, y Baptista Lucio, 2007). Se contempla como población de estudio las MYPES de los municipios de Celaya, Apaseo el Grande, Apaseo el Alto y Cortazar, las cuales conforman la totalidad de las entidades empresariales involucradas en la investigación (Bernal, 2006), mientras que la muestra se establecerá utilizando los algoritmos para poblaciones infinitas y utilizando una técnica de muestro no probabilístico por 
conveniencia debido a que por la naturaleza de la investigación es la mejor manera de elegir la muestra (Abascal, 2005).

Esta técnica es más económica con respecto a otros métodos de muestreo, además de tener la ventaja en disponibilidad para obtener la información y la factibilidad en su medición (Malhotra, 2004). La elección de la población de estudio se estableció bajo la influencia de algunos aspectos limitantes tales como la inseguridad, limitación de recursos y cercanía con la ciudad de Celaya, Gto. Para la determinación del tamaño de la muestra se utilizó el algoritmo para poblaciones infinitas, debido a que la cantidad de sujetos de investigación que conforman la población es mayor a 10000. Derivado de lo anterior, el tamaño de muestra obtenido para este estudio es de 736 sujetos de investigación, con un nivel de confianza del $97 \%(Z=2.17)$ y un error del $4 \%$, la cual se distribuyó proporcionalmente entre los sectores de manufactura, comercio y servicios. En términos prácticos solo se consideraron válidos datos de 707 unidades económicas derivado de errores en las respuestas de algunos instrumentos aplicados.

Para esta investigación se utilizó un instrumento adaptado de Padilla (2006), el cual ha sido aplicado en otras investigaciones, con validez y confiabilidad evaluada con un valor de 0.849. Dicho instrumento permitió caracterizar la cultura de innovación de las empresas estudiadas, considerando los principales parámetros identificados en la revisión de literatura.

Las opciones de respuestas de los cuestionamientos sujetos a análisis estadístico fueron de opción múltiple, aplicando escalas de Likert en los casos donde está definido un orden o nivel creciente de significancia del dato, de acuerdo con el cuestionamiento implicado, en una menor proporción hay algunas preguntas cuyas opciones de respuestas son de carácter nominal.

Para ilustrar la aplicación del instrumento documental, en la tabla 1, se presenta información de las dimensiones incluidas para caracterizar la cultura de innovación en las empresas estudiadas. 
Tabla 1 Dimensiones incorporadas en el instrumento de investigación para medir la cultura de innovación.

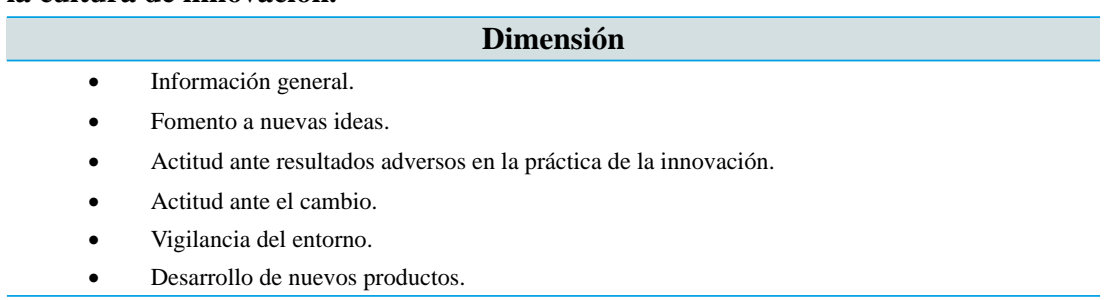

Fuente: elaboración propia (2019).

Durante la etapa de análisis de datos se recurrió a la utilización del software SPSS para capturar y procesar los datos.

\section{Resultados}

A continuación, en la tabla 2, se presentan los resultados de los ítems más relevantes en cuanto a la descripción de la cultura de innovación encontrados en la presente investigación:

Tabla 2 Resultados del instrumento de investigación aplicado para medir impacto de cultura de innovación.

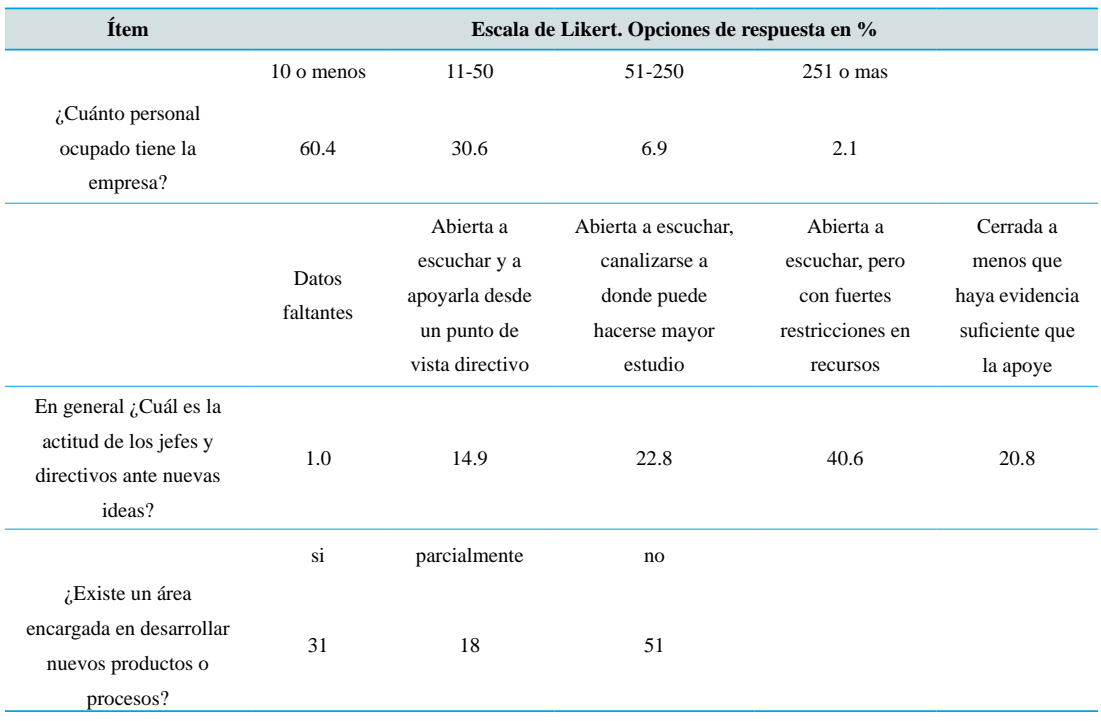

Fuente: elaboración propia (2019). 
Tabla 2 Resultados del instrumento de investigación aplicado para medir impacto de cultura de innovación (continuación).

\begin{tabular}{|c|c|c|c|c|}
\hline \multirow[t]{2}{*}{ Ítem } & \multicolumn{4}{|c|}{ Escala de Likert. Opciones de respuesta en \% } \\
\hline & $\begin{array}{c}\text { Si existen } \\
\text { formas } \\
\text { establecidas } \\
\text { y funcionan } \\
\text { adecuadamente }\end{array}$ & $\begin{array}{c}\text { Si existen formas } \\
\text { establecidas pero } \\
\text { no se les da la } \\
\text { atención debida }\end{array}$ & $\begin{array}{c}\text { No hay como tales pero las ideas } \\
\text { se canalizan cuando llegan }\end{array}$ & $\begin{array}{c}\text { No hay } \\
\text { como tales y } \\
\text { usualmente la } \\
\text { gente no sabe } \\
\text { qué hacer en } \\
\text { estos casos }\end{array}$ \\
\hline $\begin{array}{l}\text { ¿Existen medios } \\
\text { establecidos para } \\
\text { captar, evaluar y } \\
\text { desarrollar nuevas } \\
\text { ideas? }\end{array}$ & 19.8 & 18.8 & 31.7 & 29.7 \\
\hline & Dato faltante & Si, y se utiliza & Si pero opera parcialmente & $\begin{array}{l}\text { No, pero se } \\
\text { reconoce }\end{array}$ \\
\hline $\begin{array}{c}\text { ¿Hay un plan o } \\
\text { medio de incentivos } \\
\text { o promoción para } \\
\text { la gente que aporta } \\
\text { nuevas ideas? }\end{array}$ & 1 & 20 & 28 & 17 \\
\hline & Datos faltantes & $\begin{array}{l}\text { Si y funcionan } \\
\text { adecuadamente }\end{array}$ & Sí, pero no tienen mucho peso & $\begin{array}{c}\text { Si se han } \\
\text { formado pero } \\
\text { desaparecen al } \\
\text { poco tiempo }\end{array}$ \\
\hline $\begin{array}{c}\text { ¿La empresa } \\
\text { ha establecido } \\
\text { herramientas } \\
\text { orientadas a } \\
\text { la solución de } \\
\text { problemas? (grupos } \\
\text { de trabajo, análisis } \\
\text { conjunto de fallos, } \\
\text { etc.). }\end{array}$ & 1 & 28.7 & 26.7 & 19.8 \\
\hline & $\mathrm{Si}$ & Usualmente si & Generalmente no & No \\
\hline $\begin{array}{c}\text { ¿Utiliza medios } \\
\text { electrónicos para } \\
\text { mantenerse informado } \\
\text { sobre la situación de } \\
\text { los mercados, clientes, } \\
\text { competencia y otros } \\
\text { aspectos relevantes a } \\
\text { su negocio? }\end{array}$ & 45.5 & 28.7 & 15.8 & 9.9 \\
\hline
\end{tabular}

Fuente: elaboración propia (2019). 
Tabla 2 Resultados del instrumento de investigación aplicado para medir impacto de cultura de innovación (continuación).

\begin{tabular}{|c|c|c|c|c|}
\hline \multirow{2}{*}{ Ítem } & \multicolumn{4}{|c|}{ Escala de Likert. Opciones de respuesta en \% } \\
\hline & Sí & Usualmente sí & Generalmente no & No \\
\hline $\begin{array}{c}\text { ¿La empresa realiza } \\
\text { un análisis detallado } \\
\text { de los productos } \\
\text { del mercado para } \\
\text { detectar innovaciones, } \\
\text { necesidades y fallas? }\end{array}$ & 32.7 & 38.6 & 18.8 & 9.9 \\
\hline & Sí & Usualmente sí & Generalmente no & No \\
\hline $\begin{array}{c}\text { ¿La información } \\
\text { captada en el exterior } \\
\text { constituye una } \\
\text { fuente de innovación } \\
\text { importante para la } \\
\text { empresa? }\end{array}$ & 44.6 & 36.6 & 13.9 & 5.0 \\
\hline
\end{tabular}

Fuente: elaboración propia (2019).

Respecto a los resultados globales de innovación en las empresas estudiadas se muestran los valores obtenidos en la tabla 3.

Tabla 3 Media y desviación estándar global para la innovación.

\begin{tabular}{|c|c|c|c|c|}
\hline \multicolumn{5}{|c|}{ Estadísticos } \\
\hline & \multicolumn{2}{|c|}{$\mathbf{N}$} & \multirow{2}{*}{ Media } & \multirow{2}{*}{$\begin{array}{c}\text { Desviación } \\
\text { estándar }\end{array}$} \\
\hline & Válido & Perdidos & & \\
\hline Innovación & 707 & 0 & 2.1337 & 68948 \\
\hline
\end{tabular}

Fuente: elaboración propia (2019). 
Se ratifica a través de la investigación bibliográfica que las MYPES son el tipo de empresas consideradas el motor de la economía no solo de la región, sino a nivel nacional e internacional y son la base del sustento económico y ocupacional de muchas familias, representando en promedio en algunos países hasta el 90\% de todas las unidades económicas (Ponce y Ceballos, 2017), y en México, las PYMES representan una generación de empleo del 42\% (Gil y Jiménez, 2014).

Guanajuato es uno de los estados donde existen mayor cantidad de MYPES de todo el país, de ahí la importancia que tienen dichas empresas por su contribución al desarrollo económico estatal y, de este modo generar investigación que contribuya al desarrollo de dichas empresas.

A partir de los datos encontrados y descritos por la tabla 2, se puede inferir que el personal ocupado que tiene la empresa en promedio es de 3.46 empleados. La antigüedad promedio de estar laborando en las empresas es de 9.63 años con una desviación estándar de 10 años, lo cual nos indica una variabilidad significativa de años laborados entre personas con menos o más de 10 años arriba o debajo del promedio. El tiempo de operación promedio de las empresas es de 9.86 años.

En cuanto a los cuestionamientos más destacables que tiene que ver con la cultura de innovación se encontró que en un $40 \%$ de las empresas estudiadas, la actitud de los jefes y directivos ante las nuevas ideas en promedio es abierta a escucharlas, pero con fuertes restricciones en recursos para llevarlas a cabo, en un $22.8 \%$ de empresas se escuchan las ideas, pero canaliza a donde puedan hacerse mayores estudios. En un 31\% de las organizaciones sí existe un área para desarrollar nuevos productos o procesos, mientras que en un $18 \%$ solo existe parcialmente y en un 51\% no hay un área exclusiva para dicho fin.

En el 19.8 \% de las empresas encuestadas existen formas establecidas para captar, evaluar y desarrollar nuevas ideas y funcionan adecuadamente. En un 18.8\% existen dichas formas, pero no se les da la atención debida y, en otras organizaciones, no hay medios como tales, pero las ideas se canalizan cuando llegan. 


\section{Discusión}

Cuando se presentan nuevas ideas en la mejora de procesos internos, en un $46.6 \%$ de las empresas, la actitud del personal es de interés hacia ellas. Por otra parte, en un 56.4\% de las organizaciones la actitud del personal es de indiferencia, escepticismo o rechazo.

El promedio de empresas mencionó que sí existe un plan o medio de incentivos o promoción para la gente que aporta nuevas ideas, pero que opera parcialmente.

En un $37.8 \%$ de las empresas encuestadas existen registros de marca, en un $21.1 \%$ existen patentes, en el $14.4 \%$ diseños industriales y en un $10 \%$ modelos de utilidad.

En un 28.7 \% de las empresas sí se han establecido herramientas orientadas a la solución de problemas y funcionan adecuadamente, aunque en un 26.7 \% están establecidas, pero no tienen mucho peso en el funcionamiento de la misma. Las empresas en promedio, mencionaron que usualmente sí utilizan medios electrónicos para mantenerse informadas sobre la situación de los mercados, clientes, competencia y otros aspectos relevantes para sus negocios. Estas empresas en promedio sí realizan un análisis detallado de los productos del mercado para detectar innovaciones, necesidades y fallas.

Las empresas están abiertas a modificar sus creencias y conductas si aparece una tecnología revolucionaria y estratégica para el futuro de la empresa. La información captada en el exterior constituye una fuente de innovación importante para la empresa. En las empresas existe un plan a largo plazo sobre la evolución comercial de la empresa, pero no se ha formalizado para su implementación.

En la tabla 3, se presentan los resultados generales encontrados en la variable innovación. Se observa que el valor esperado de la variable innovación es de 2.1 y con una desviación estándar de 0.68 lo cual nos indica que la mayoría de las empresas que participaron en la investigación poseen un nivel de innovación de bajo a moderado 
tomando como base que 1 representa un nivel bajo, 2 representa un nivel de bajo a moderado, 3 equivale a un nivel de moderado a alto y 4 significa un nivel alto.

En la figura 2, se describe que la moda de la variable innovación es de 2.0 lo cual indica que la mayoría de las empresas que participaron en la investigación poseen un nivel de innovación de bajo a moderado tomando como base que 1 representan un nivel bajo, 2 representa un nivel de bajo a moderado, 3 equivale a un nivel de moderado a alto y 4 significa un nivel alto.

En la figura 3, se muestran los niveles de cultura de innovación encontrados en cada una de las ciudades que conformaron la zona de estudio, observándose que la ciudad de Apaseo el Grande es la que posee el nivel más elevado de innovación (2.4), seguido por Celaya, Cortazar y finalmente la ciudad de Apaseo el Alto con el nivel más bajo.

En la figura 4, se observa que el sector manufactura presenta, el nivel más elevado

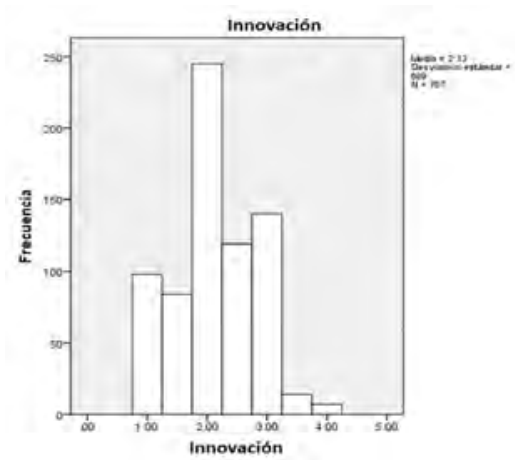

Figura 2. Resultados globales de la innovación.

Fuente: elaboración propia (2019).

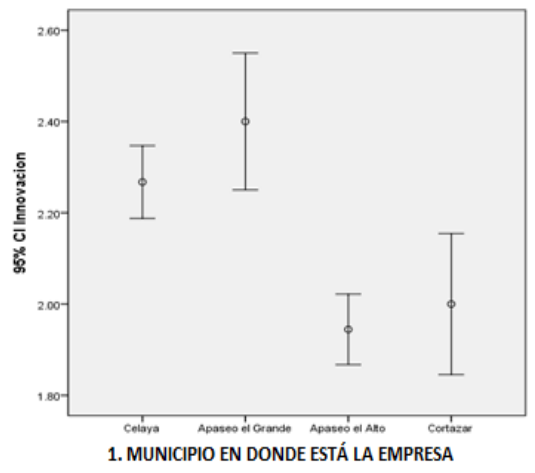

Figura 3. Niveles de cultura de innovación por ciudad.

Fuente: elaboración propia (2019). 


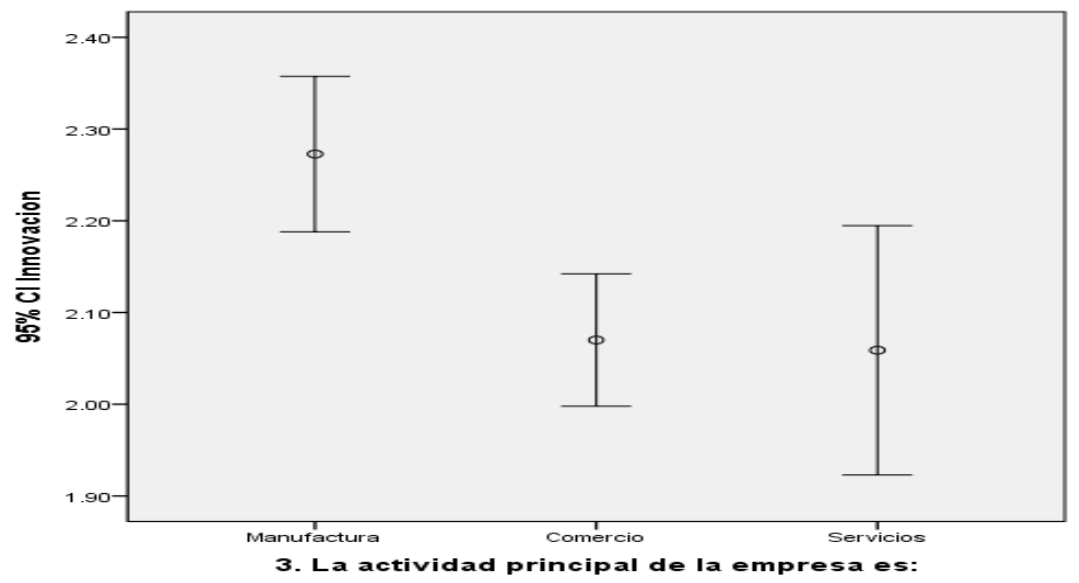

Figura 4. Niveles de cultura de innovación por sector.

Fuente: elaboración propia (2019).

de innovación (2.27); en contraste con los sectores de comercio y servicios (2.06 y 2.05 respectivamente), teniendo este último sector mayor variabilidad dentro de sus empresas.

\section{Conclusión}

Se identificaron características y variables de cultura de innovación en las micro y pequeñas empresas estudiadas, pertenecientes a los municipios de Celaya, Cortazar, Apaseo el Alto y Apaseo el grande en niveles relativamente aceptables, aunque no los idóneos para alcanzar los estándares de calidad, productividad y competitividad que requieren este tipo de organizaciones hoy en día. Sería deseable que los líderes y directivos de las empresas conozcan y adquieran los valores de la cultura de innovación, para que ellos transmitan e inculquen en todo el personal dichos principios. Es necesario difundir el conocimiento de que el valor que posee la innovación en productos, servicios, procesos y comercialización, es generar productividad, competitividad y satisfacción personal y empresarial. De esta manera las organizaciones podrían mejorar 
su desempeño, y el nivel de bienestar de los habitantes de este país, en el marco de un mundo globalizado. Es importante continuar investigando este tipo de empresas, debido a que contribuyen de manera importante con la generación de empleo.

Se espera que los resultados de esta investigación contribuyan a una mejor toma de decisiones por parte de las autoridades gubernamentales, específicamente a las direcciones de desarrollo económico estatales y de las mismas empresas, para la generación de políticas públicas y privadas, que incrementen el desarrollo de las MYPES.

\section{Referencias bibliográficas}

Abascal, E. (2005). Análisis de encuestas. Madrid: ESIC Editorial.

Andrade, J., Gasca, A., Perdomo, A., Benavides, D. y Sánchez, H. (2019). El capital intelectual en asociaciones de productores del sur de Colombia. Tendencias en la Investigación Universitaria Una visión desde Latinoamérica. Vol. VI Capitulo XIII. 187-201. Recuperado de https://investigacionuptag. wordpress.com/www.alinin.org.

Bernal, C. (2006). Metodología de la investigación: para administración, economía,humanidades y ciencias sociales. México: Pearson Educación.

Carbache, O., Frías, C., Gutiérrez, G. y Véliz, G. (2017). La cultura organizacional parala innovación y el desarrollo competitivo de las pequeñas y medianas empresas (pymes) exportadoras de las guayas. Revista Empresarial, ICE-FEE-UCSG, 11(3). Recuperado de http://webcache. googleusercontent.com/search?q=cache:qGjSUrKoqJQJ:editorial. ucsg.edu.ec/ojs-empresarial/index.php/empresarial-ucsg/article/ download/98/87+\&cd=3\&hl=es\&ct=clnk\&gl=mx.

Gil, A.A. y Jiménez, J. J. (2014). El contexto económico global de la Pyme”. Revista Académica e Institucional, 95, 155-179. Recuperado de file://C:/ Users/Dell/Downloads/Dialnet-ElContextoEconomicoGlobalDeLaPY ME-5213948\%20(1).pdf. 
Gomes, G., y Wojahn, R. (2017). Organizational learning capability, innovation and performance: study insmall and medium-sized enterprises (SMES). Revista de Administração, 52, 163-175. Recuperado de http://www.sciencedirect. com/science/article/pii/S0080210716308287.

Góngora, J. (2013). El panorama de las micro, pequeñas y medianas empresas en México. Comercio Exterior, 63(6), 2-6. Recuperado de http://revistas. bancomext.gob.mx/rce/magazines/754/1/El_paronama_de_las_micros.pdf.

Guerra, P. (2010). Estudio Sobre la Percepción en Innovación y Desarrollo entre los Administradores de las Pequeñas y Medianas Empresas: Caso de Estudio Monterrey, Nuevo León. Daena: International Journal of Good Conscience. 5(2), 246-276. Recuperado de. http://www.spentamexico.org/ v5-n2/5(2)246-276.pdf

Hernández, R., Fernández, C. y Baptista, M. (2007). Metodología de la investigación (4ta ed.). México: Mc Graw Hill.

Islam, A. \& Tedford, D. (2012). Risk determinants of small and medium-sized manufacturing enterprises (SMEs) - an exploratory study in New Zealand. Journal of Industrial Engineering International, 8(12), 1-13. Recuperado de http://www.jiei-tsb.com/content/8/1/12.

Maldonado, G., Ojeda, J., Uc. L., Valdez, L. y Medina, M. (2017). La microempresa en México: Un diagóstico de su situación actual. México: Universidad Autónoma de Aguascalientes. Recuperado de https://www.uaa.mx/ direcciones/dgdv/editorial/docs/ve_microempresaenmexico.pdf.

Malhotra, N. (2004). Investigación de mercados: un enfoque aplicado. México: Pearson Educación.

Molina, R. y Contreras, R. (2016). La metropolización de la región Laja Bajío como alternativa para abatir la desigualdad social y generar competitividad sustentable. Universidad de Guanajuato. 3 (3). Recuperado de https:// www.academia.edu/28026285/La_metropolizaci\%C3\%B3n_de_la_ regi\%C3\%B3n_Laja-Baj\%C3\%ADo. 
Padilla, R. (2006). Instrumentos de medición de la competitividad. Por la Comisión Económica para América Latina y el Caribe (CEPAL) Sede Subregional México. Recuperado de https://goo.gl/cRazLl.

Ponce, F. y Zevallos, E. (2017). La innovación en la micro y la pequeña empresa (MYPE): no solo factible, sino accessible. I Congreso Internacional de Ciencias de la Gestión: Gestión de la Innovación e Innovación en la Gestión Pontificia Universidad Católica del Perú. Facultad Latinoamericana de Ciencias Sociales. 46-68. Recuperado de file://C:/Users/hp/Downloads/1905075633-1-PB.pdf.

Putthiwanit, C. (2015). Exploring the impact of organizational culture on employees in multinational enterprise: A qualitative approach. Procedia - Social and Behavioral Sciences, 207, 483 - 491. Recuperado de http://www.sciencedirect.com/. hiwanit \&pub $=\&$ volume $=\&$ issue $=\&$ page $=\&$ origin $=$ home $\&$ zone $=\mathrm{qSearch}$.

Robbins, S., y Coulter, M. (2014). Administración. México: Pearson.

Rodríguez, J. (2010). Administración de pequeñas y medianas empresas. México: CENGAGE learning.

Rueda, G., y Rodenes, M. (2010). Hacia una cultura innovadora en las empresas. Universidad Pontificia Bolivariana y Universidad Politécnica de Valencia, España,1-12. Recuperado de https://www.researchgate.net/ publication/267213263.

Sampedro, J.L., y Díaz, C. (2016). Innovación para el desarrollo inclusivo: Una propuesta para su análisis. Economía Informa. 396, 34-48. Recuperado de http://www. economia.unam.mx/assets/pdfs/econinfo/396/02SampedroDiaz.pdf.

Torres T, González A. y Cano A. (2019). Procesos de innovación tecnológica en grupos empresariales. El caso de una empresa de autoservicios. Tendencias en la investigación universitaria Vol. VI (Una visión desde Latinoamérica) Capítulo X. 145-158. Recuperado de https://investigacionuptag.wordpress. $\mathrm{com} / \mathrm{www}$.alinin.org. 